\title{
Comoving Distance- Light Travel Distance (Treatise)
}

\author{
Weitter Duckss \\ Independent Researcher, Zadar, Croatia \\ wduckss@gmail.com
}

\begin{abstract}
The discussion on the values of redshift, as well as blueshift, is based on a large increase in new evidence that in the whole volume of Universe there are gravitationally-bound objects (galaxies, clusters and super clusters of galaxies) „Using the Chandra and Hubble Space Telescopes we have now observed 72 collisions between galaxy clusters, including both 'major' and 'minor' mergers" (Harvey, 2015). That adds to a great diversity of galactic movement directions and their diverse appearances to an observer. The accent here is at the point of "clearing the early Universe" and asks questions about how these types of radiation could be measured if all galaxies were created in the early stages of Universe, which had started emitting these types of radiation. At the same time, some questions are asked about. With a redshift of 5.47, (Q0906 + 6930) light from this active galaxy is estimated to have taken around 12.3 billion light-years to reach us.. distance to this galaxy is estimated to be around 26 billion light-years (7961 Mpc). (NASA/IPAC, 2010) i.e., why the actual measured values of redshift are not applied.
\end{abstract}

Keywords: comoving distance; light travel distance

\section{Introduction}

The article deals with the newest measured data for the most distant galaxies with a significant redshift, for which there are two values, co-moving distance - light travel distance.

A special attention is dedicated to the difference between a value of redshift and the transformation into a distance below 13, 8 Gly, distance 28.85 Gly (8.85 Gpc) (co-moving); 12.9 Gly (4.0 Gpc) (light travel distance ULAS J1120+0641).

As a starting point of this discussion I use the mainstream claims that the first types of radiation originated $320.000-380.000$ years after a hypothetical beginning or creation of Universe and the spreading speed of Universe is always lower than the spreading speed of radiation (waves).

The method of verification is the usage of a sequence of relations with mainstream evidence in a single place to eliminate the possibility of manipulation with data and conclusions. To speed up the release of this article, I will use tables and photographies already published in my articles (Duckss, 2018), (Duckss, 2018), (Duckss, 2019), (Duckss, 2019) and used in discussing other topics.

\section{Review of Literatures}

\section{Values of Blueshift and Redshift Presentation}

For already some time science faces the problem of redshift value, which is determined very differently at the same distances; also, for the same value of redshift (z) there are very different distances and the speeds of withdrawal from (and approaching to) an observer, in the whole volume of Universe. Nowadays, as well as in the early stages of discovering new galaxies, the existence of blueshift has been ascribed and related only to the local group. There is a similar quantity of galaxies with redshift and blueshift in our local group. 
Table 1. Our Local Galaxy Group (Part)

\begin{tabular}{|c|c|c|}
\hline Galaxies, Local Groups & Redshift km/s & Blueshift km/s \\
\hline Sextans B $(4.44 \pm 0.23 \mathrm{Mly})$ & $300 \pm 0$ & \\
\hline Sextans A & $324 \pm 2$ & \\
\hline NGC 3109 & $403 \pm 1$ & \\
\hline Tucana Dwarf & $130 \pm ?$ & \\
\hline Leo I & $285 \pm 2$ & \\
\hline NGC 6822 & & $-57 \pm 2$ \\
\hline Andromeda Galaxy & & $-301 \pm 1$ \\
\hline Leo II (about 690,000 ly) & $79 \pm 1$ & \\
\hline Phoenix Dwarf & $60 \pm 30$ & \\
\hline SagDIG & & $-79 \pm 1$ \\
\hline Aquarius Dwarf & & $-141 \pm 2$ \\
\hline Wolf-Lundmark-Melotte & & $-122 \pm 2$ \\
\hline Pisces Dwarf & & $-287 \pm 0$ \\
\hline Antlia Dwarf & $362 \pm 0$ & \\
\hline Leo A & 0.000067 & \\
\hline Pegasus Dwarf Spheroidal & & $-354 \pm 3$ \\
\hline IC 10 & & $-348 \pm 1$ \\
\hline NGC 185 & & $-202 \pm 3$ \\
\hline Canes Venatici I & $\sim 31$ & \\
\hline Andromeda III & & $-351 \pm 9$ \\
\hline Andromeda II & & $-188 \pm 3$ \\
\hline Triangulum Galaxy & & $-179 \pm 3$ \\
\hline Messier 110 & & $-241 \pm 3$ \\
\hline NGC $147(2.53 \pm 0.11 \mathrm{Mly})$ & & $-193 \pm 3$ \\
\hline Small Magellanic Cloud & 0.000527 & \\
\hline M32 & & $-200 \pm 6$ \\
\hline NGC 205 & & $-241 \pm 3$ \\
\hline IC 1613 & & $-234 \pm 1$ \\
\hline Carina Dwarf & $230 \pm 60$ & \\
\hline Sextans Dwarf & $224 \pm 2$ & \\
\hline Ursa Minor Dwarf $(200 \pm 30 \mathrm{kly})$ & & $-247 \pm 1$ \\
\hline Draco Dwarf & & $-292 \pm 21$ \\
\hline Cassiopeia Dwarf & & $-307 \pm 2$ \\
\hline Ursa Major II Dwarf & & -116 \\
\hline Leo IV & 130 & \\
\hline Leo V ( $585 \mathrm{kly})$ & 173 & \\
\hline Leo $\mathrm{T}$ & & -60 \\
\hline Bootes II & & -120 \\
\hline Pegasus Dwarf & & $-183 \pm 0$ \\
\hline Sculptor Dwarf & $110 \pm 1$ & \\
\hline Etc. & & \\
\hline
\end{tabular}

Table 1. Our Local Galaxy Group (Part) with redshift and blue shift km/s (Duckss, 2018). 
As technology advances and the quantity of the observed galaxies increases, many galaxies with blueshift (i.e., those that approach to an observer) outside our local group were discovered. All those galaxies that are gravitationally-bound (large and small mergers, the collision of galaxies - interactive galaxies) are to be added to those galaxies having spectral blueshift. Although we, the observers, notice their more or less expressed redshift, all of these galaxies exclusively experience blueshift between each other, due to approaching or collision. Recent research have discovered 200.000,0 galaxies in the interaction. [7]

Table 2. A Part of Galaxies with Blueshift

\begin{tabular}{|c|c|c|c|}
\hline Designation & VLG... blue shift & Designation & VLG... blue shift \\
\hline IC3036 & -126 & NGC4419 & -383 \\
\hline IC3044 & -298 & VCC997 & -360 \\
\hline VCC087 & -267 & KDG132 & -100 \\
\hline NGC4192 & -246 & NGC4438 & -43 \\
\hline NGC4212 & -199 & SDSS & -0 \\
\hline VCC181 & -267 & VCC1129 & -105 \\
\hline VCC200 & -98 & VCC1163 & -564 \\
\hline A224385 & -204 & VCC1175 & -118 \\
\hline IC3094 & -275 & VCC1198 & -470 \\
\hline VCC237 & -423 & IC3416 & -198 \\
\hline IC3105 & -284 & VCC1239 & -672 \\
\hline VCC322 & -323 & VCC1264 & -539 \\
\hline VCC334 & -350 & IC3435 & -150 \\
\hline VCC501 & -224 & VCC1314 & -37 \\
\hline IC3224 & -100 & IC3445 & -470 \\
\hline VCC628 & -540 & IC3471 & -235 \\
\hline VCC636 & -113 & IC3476 & -280 \\
\hline IC3258 & -593 & IC3492 & -604 \\
\hline IC3303 & -427 & IC3548 & -37 \\
\hline VCC788 & -3 & VCC1682 & -66 \\
\hline VCC802 & -318 & NGC4569 & -345 \\
\hline IC3311 & -287 & UGC7795 & -78 \\
\hline VCC810 & -470 & VCC1750 & -258 \\
\hline VCC815 & -866 & VCC1761 & -269 \\
\hline VCC846 & -845 & KDG172 & -42 \\
\hline NGC4396 & -215 & VCC1812 & -351 \\
\hline VCC877 & -212 & VCC1860 & -124 \\
\hline NGC4406 & -374 & IC3658 & -69 \\
\hline VCC892 & -784 & UGC7857 & -7 \\
\hline NGC4413 & -16 & VCC1909 & -16 \\
\hline VCC928 & -395 & IC0810 & -188 \\
\hline IC3355 & -126 & VCC2028 & -52 \\
\hline VCC953 & -563 & & \\
\hline
\end{tabular}

Table 2. A part of galaxies with blueshift (and negative speeds) at the distance of about $53.8 \pm$ 0.3 Mly (16.5 $\pm 0.1 \mathrm{Mpc})$. [8]

At the distances above 70 Mly the values of (mostly) blueshift or approaching (the galaxies seen from Earth) are annihilated, also due to the increase in distance, which is the 
reason to decrease the intensity (force) of waves (radiation). Above 70 Mly our instruments read redshift, regardless of approaching or withdrawing of an object from an observer.

„The digital twin is used in association with the physical throughout the life cycle: during the testing, rework, operation and disposal phase. The physical object uses sensors that collectreal-time data about the state of the object, after which this information is sent to the digital“(Duckss, 2019)

However, at the distances above 70 Mly and below them

(,NGC 1.600 is 149,3 Kly away and its speed is $4.681 \mathrm{~km} / \mathrm{s}$,

NGC 7320c is 35 Mly away and with the speed of (a red shift) $5.985 \pm 9$,

NGC 5010 that is 469 Mly away has the speed of distancing of $2.975 \pm 27$, and the galaxy

NGC 280 that is 469 Mly away has the speed of distancing of 3.878 !

At the distance of $52 \pm 3$ (M86) there is a blue shift $(-244 \pm 5 \mathrm{~km} / \mathrm{s})$ that is also present with the galaxy M90 at the distance of $58.7 \pm 2.8(-282 \pm 4)$, while the other galaxies at the same distance (Messier 61, NGC 4216, Messier 60, NGC 4526, Messier 99 (except NGC 4419 $-0,0009(-342))$ are with a positive sign and completely different speeds." (Duckss, 2018)). Wecan read different values of redshift for the same distances or the same redshift value for the galaxies that have very different distances.

Table 3. Red Shift /Distance

\begin{tabular}{|c|c|c|c|}
\hline & Galaxy, Cluster galaxy, Supercluster & Red shift (z) & Distance M ly \\
\hline 1 & Leo_Cluster & 0,022 & 368,6 \\
\hline$\underline{2}$ & ARP 87 & $\underline{0,023726}$ & 330 \\
\hline$\overline{3}$ & Abell 2152 & $\overline{0,041}$ & 551 \\
\hline 4 & $\overline{\text { Hydra_Cluster }}$ & 0,0548 & 190,1 \\
\hline 1 & Abell 671 & 0,0502 & 600 \\
\hline 2 & Abell 1060 & 0,0548 & 190,1 \\
\hline 3 & Abell 1991 & 0,0587 & 812 \\
\hline 4 & Corona Borealis Supercluster & 0,07 & 946 \\
\hline 5 & Laniakea Supercluster & 0,0708 & 250 \\
\hline 6 & Abell 2029 & 0,0767 & 1063 \\
\hline 1 & $\overline{\text { Abell } 383}$ & 0,1871 & 2485 \\
\hline 2 & Abell 520 & 0,2 & 2645 \\
\hline 3 & Abell_222(3) & 0,211 & 2400 \\
\hline 1 & Saraswati Supercluster & 0,28 & 4000 \\
\hline 2 & Bullet Cluster & 0,296 & 3700 \\
\hline 3 & Abell 2744 & 0,308 & 3982 \\
\hline 4 & $\overline{\text { CID-42 }}$ & 0,359 & 3900 \\
\hline 1 & Abell 370 & 0,375 & 4775 \\
\hline 2 & 3 C 295 & 0,464 & 4600 \\
\hline$\underline{3}$ & Musket Ball Cluster & 0,53 & 700 \\
\hline$\overline{4}$ & Abell 754 & 0,542 & 760 \\
\hline$\underline{1}$ & MACS J0025.4-1222 & $\underline{0,586}$ & 6070 \\
\hline$\underline{2}$ & Phoenix Cluster & $\overline{0,597}$ & 5700 \\
\hline$\underline{3}$ & $\overline{\mathrm{RX} \mathrm{J1131-1231}}$ & $\underline{0,658}$ & 6050 \\
\hline 4 & ACT-CL J0102-4915 & 0,87 & 4000 \\
\hline 1 & $\overline{\text { Lynx Supercluster }}$ & $\overline{1,26}, 1,27$ & 12000 \\
\hline 2 & Twin Quasar & 1,413 & 8700 \\
\hline 3 & XMMXCS_2215-1738 & 1,45 & 10000 \\
\hline
\end{tabular}




\begin{tabular}{|l|l|l|l|}
\hline 4 & Einstein Cross & 1,695 & 8000 \\
\hline 5 & TON 618 & 2,219 & 10400 \\
\hline 6 & EQ J100054+023435 & $\underline{4,547}$ & 12200 \\
\hline 7 & Q0906+6930 & 5,47 & 12300 \\
\hline 1 & $\underline{\text { z8 GND 5296 }}$ & $7,5078 \pm 0,0004$ & 13100 \\
\hline 2 & $\underline{\text { A2744 YD4 }}$ & 8,38 & 13200 \\
\hline 3 & $\underline{\text { UDFy-38135539 }}$ & 8,6 & 13100 \\
\hline 4 & GRB 090429B & 9,4 & 13140 \\
\hline 5 & $\underline{\text { Abell 1835 IR1916 }}$ & 10,0 & 13200 \\
\hline
\end{tabular}

Table 3. As redshift increases, the distance of the objects decreases, increases (faster or slower than "expected") or remains similar. (Duckss, 2019) It is very well known in science that the intensity of radiation decreases due to the increase of distance (which is obvious when at night we look at the stars with bare eyes).

„The pressure of the electromagnetic radiation, measured in $\mu \mathrm{Pa}\left(\mu \mathrm{N} / \mathrm{m}^{2}\right.$ and $\left.\mathrm{N} / \mathrm{km}^{2}\right)$, is as follows: 915, on the distance of $0.10 \mathrm{AU}$ (astronomical units) away from Sun; 43.3 on Mercury; 9.15 on Earth; 0.34 on Jupiter. Or, measured in pound-force per square miles $\left(\mathrm{lbf} / \mathrm{mi}^{2}\right)$ : 526, 0.10 AU away from Sun; 24.9 on Mercury: 5.26 on Earth; 0.19 on Jupiter.

The average intensity of the solar radiation, in W/ $\mathrm{m}^{2}$, is as follows: 9116.4 on Mercury; 1366.1 on Earth; 50.5 on Jupiter; 0.878 on Pluto. Wikipedia „The interaction of space and radiation directly influences the temperature of an object. On the following objects' surfaces it is as follows: $440^{\circ} \mathrm{K}$ on Mercury; $288^{\circ} \mathrm{K}$ on Earth; 152 on Jupiter 16 . The space around the objects has the same decreasing curve starting from the Sun towards the end of the system. The same goes for the dark side of the objects. The lowest temperature on Mercury is $100^{\circ} \mathrm{K}$, on Uranus $49^{\circ} \mathrm{K}$, on Pluto $28^{\circ} \mathrm{K}$, in the Oort cloud $4^{\circ} \mathrm{K}$. During observation, a compensation for the atmospheric influence and the interior temperature of an object needs to be taken into consideration, as these are the factors of interference when comparative data are being acquired“(Duckss, 2016).

Although this is common knowledge, astronomers do not apply it in determining the real distance of objects that are more or less distant in the volume of Universe and the increase of redshift is related only to the increase of distance (so-called speed of galaxies' withdrawal from an observer). The evidence mentioned above, which encompass the whole - reachable with modern instruments - volume of Universe, point out that the increase of redshift is directly related to the decrease of the measured intensity of incoming waves and its value is corrected, depending on whether an object withdraws or approaches an observer. If these two values are applied, then the confusion that was created when applying only the increase of speed with the objects withdrawing further from us disappears.

„The probability density functions of the minimum and maximum order statistics of the transmuted logistic distribution are established and the relationships between the probability density functions of the minimum and maximum order statistics of the parent model and the probability density function of the transmuted logistic distribution are considered. The parameter estimation is done by the method of maximum likelihood estimation.

The digital twin allows reproduce all other indicators of the object by minimum key parameters. With this technology it is possible to solve various classes of tasks of diagnostics of object state, forecasting, optimization of operation, control.“" (Samuel, 2019)

It has to be stated clearly that, due to this concept, we have neither realistic values of positioning the distances of objects nor the observed volume of Universe. The further the distance, the weaker are the radiation, while redshift increases and is not limited to predetermined fixed constructions that do not allow a realistic overview of Universe. 


\section{Discussion}

\section{Comoving Distance- Light Travel Distance}

We are going to check the reality of these parameters, which are strictly imposed to scientists, from the angle of very limiting factors, presented nowadays by the scientific mainstream, and convince ourselves in credibility of their application and the validity of results.

If all theories are excluded, picture 1 should approximately present real values of measurement of objects in Universe (as well as those objects that are going to be discovered in near future, due to the ongoing progress of technology).

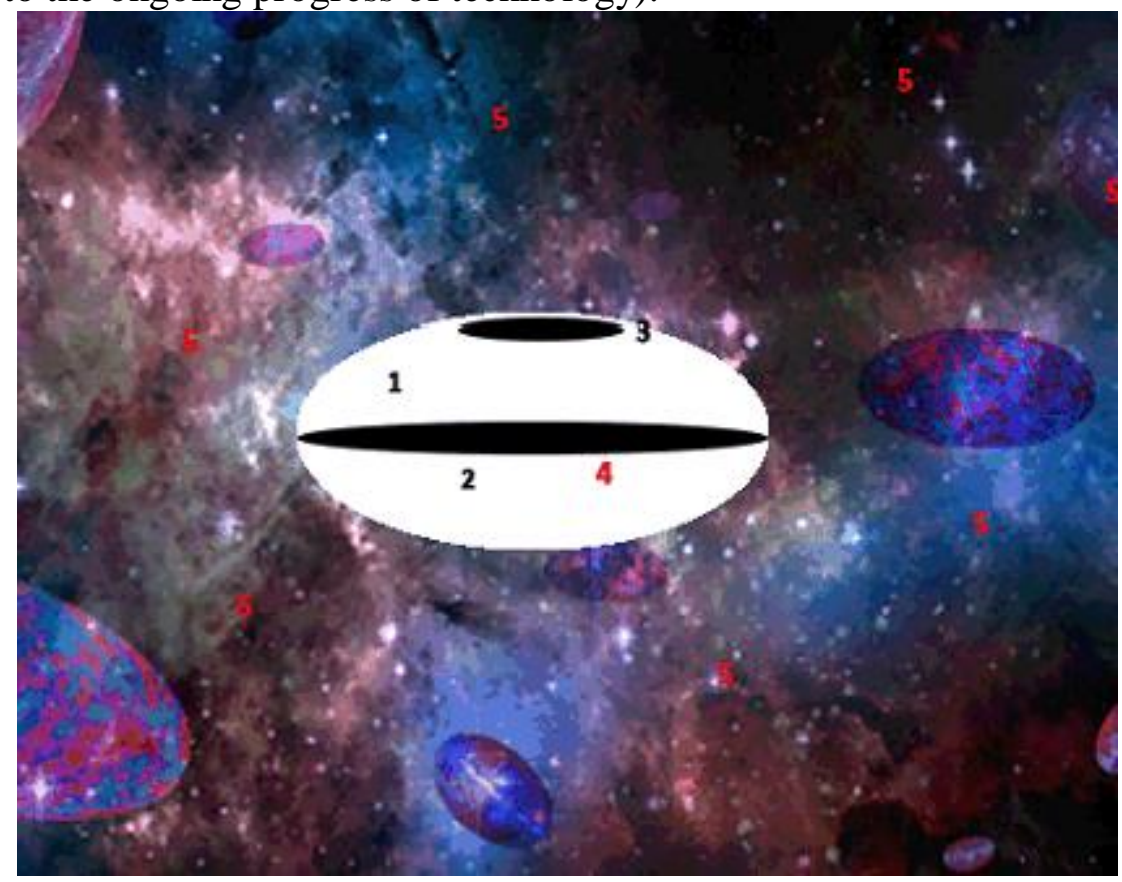

Figure 1. Universe with the points from 1-4 and its maximum possible diameter of 13,8 Gly

Mainstream science claims that 13,8 Gly is a total value of Universe, regardless of simultaneous evidence (claims).

„The proper distance for a redshift of 8.2 would be about $9.2 \mathrm{Gpc}$, or about 30 billion light years."

„With a redshift of 5.47,(Harvey, 2015)(NASA/IPAC , 2010) light from this active galaxy is estimated to have taken around 12.3 billion light-years to reach us.. distance to this galaxy is estimated to be around 26 billion light-years $(7961 \mathrm{Mpc})$ and data from published measurements: (Attila, 2009)

RD1

With a redshift of 5.34, light from this galaxy is estimated to have taken around 12.5 billion years to reach us. But since this galaxy is receding from Earth, the present comoving distance is estimated to be around 26 billion light-years.

ULAS J1120+0641

(at a comoving distance of 28.85 billion light-years) was the first quasar discovered beyond a redshift of 7.)

UDFj-39546284

Subsequently it was reported (December 2012) to possibly be at a record-breaking redshift $\mathrm{z}=11.9$ using Hubble and Spitzer telescope data, including Hubble Ultra-Deep Field (HUDF).

UDFy-38135539 
The light travel distance of the light that we observe from UDFy-38135539 (HUF.YD3) is more than 4 billion parsecs (13.1 billion light years), and it has a luminosity distance of 86.9 billion parsecs (about 283 billion light years).

There are a number of different distance measures in cosmology, and both "light travel distance" and "luminosity distance" are different from the comoving distance or "proper distance" generally used in defining the size of the observable universe[16][17] (comoving distance and proper distance are defined to be equal at the present cosmological time, so they can be used interchangeably when talking about the distance to an object at present, but proper distance increases with time due to the expansion of the universe, and is the distance used in Hubble's law.

EGS-zs8-1

The galaxy has a comoving distance (light travel distance multiplied by the Hubble constant, caused by the metric expansion of space) of about 30 billion light years from Earth.

\section{Z8 GND 5296}

Due to the expansion of the universe, this position is now at about 30 billion light-years $(9.2 \mathrm{Gpc})$ (comoving distance) from Earth.

Q0906 + 6930

But since this galaxy is receding from Earth at an estimated rate of 285,803 km/s(Harvey, 2015) (the speed of light is $299,792 \mathrm{~km} / \mathrm{s}$ ), the present (co-moving) distance to this galaxy is estimated to be around 26 billion light-years (7961 Mpc).

GN-108036

The redshift was $\mathrm{z}=7.2$, meaning the light of the galaxy took 12.9 billion years to reach Earth and therefore its formation dates back to 750 million years after the Big Bang . Redshift $\mathrm{z}=7.213$.

The existence of redshift above the value (z) 5 pointed out that if $(\mathrm{z})$ continues to grow, the concept of mainstream - 13,8 Gly (Big Bang) - is going to fall apart. Nowadays, the instruments register the value $(\mathrm{z})$ of 11,9 . When there is an overwhelming resistence from reactionary institutions and scientists, despite the newest measurements, then there appear unbelievable new ideas that do not belong to physics nor they represent science. The theme that is discussed here is one of them.

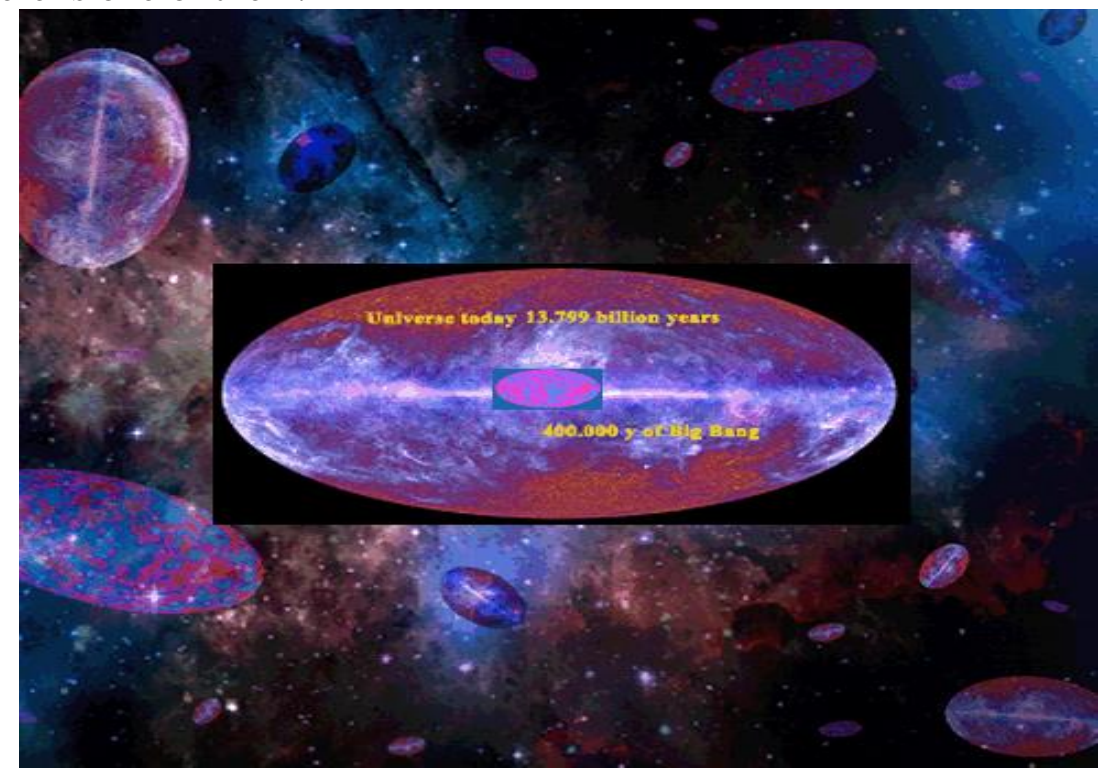

Figure 2. The Expanding Universe - history (my compilation) 
If there was a Big Bang, all the waves from that time should be approaching from a single direction, as shown in the figure 2. If radiation started for the first time 320000 - 380000 years after the explosion, during the so-called period of clearing the compact thick mass, then that radiation is impossible to measure today, no matter what mathemathical method may be used in the process. The reason to it is that all galaxies are created inside that mass that started emitting radiation. Since mainstram science also disagrees with the idea that the expansion of Universe or matter movement was faster than the spreading speed of waves in the space (which is still dubbed vacuum by the same mainstream), it can be seen that so-called measurements from that time are impossible to have been done.

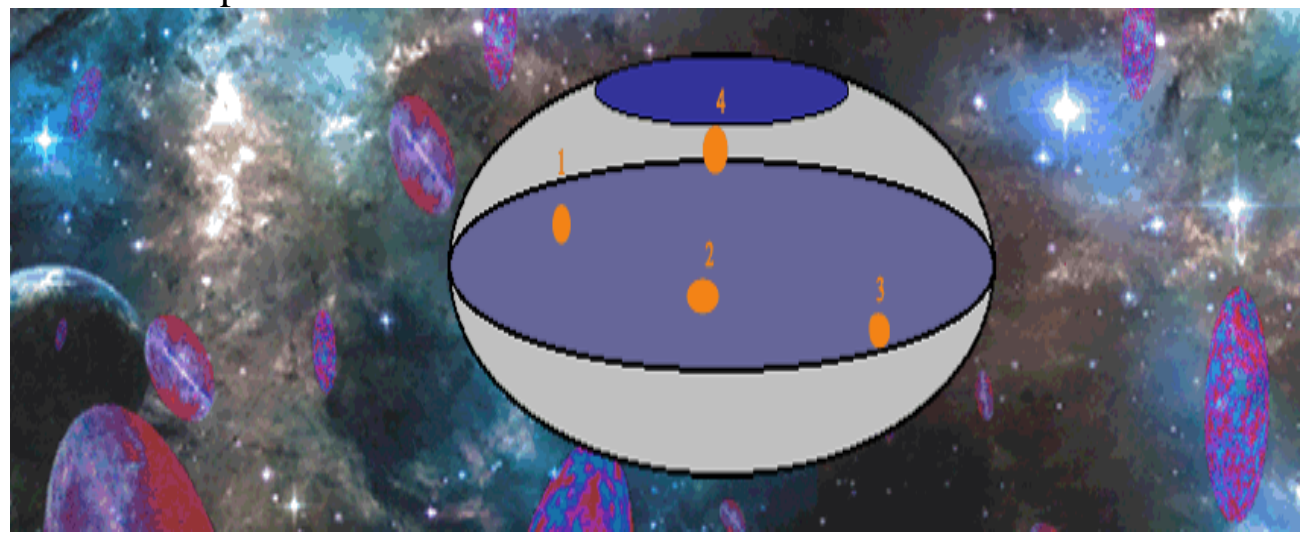

Figure 3. The Early Universe 320,000-380,000 years after the Big Bang, points 1-4 of Milky Way

We measure the objects, the age of which is estimated by mainstream to be withdrawn into past approximately as far as to the so-called early Universe, when the emission of radiation started. Points 1-4 in the so-called early Universe are some of the positions where our galaxy originated (Milky Way: $13.8 \pm 4$ billion years is age for $\mathrm{BD}+17^{\circ} 3248$; about 13.5 billionyears-old, 2MASS J18082002-5104378 B..).

Within the most distant galaxies must be objects of similar age. Milky Way has a redshift (z) 0 , the outermost galaxies have a redshift (z) 11.

Early Universe is also related to a small diameter, because the expansion has been taking place during all 13,8 Gly, due to which a contemporary volume of Universe should be created. If it was true that this small diameter of Universe started emitting radiation, besides the need for it to arrive from a single direction, it would have been obvious that this radiation left socalled early Universe with a diameter of only four times the diameter of our galaxy (under the condition that the expansion had been taking place at the speed of light). The universe has about 100 billion galaxies.

The deepest radiation of the early Universe needed to travel through only 200.000 ly in order to leave our Universe. The other problem is that the mainstream claims that Universe spreads ever faster, because the most distant galaxies show the most important redshift.

However, it is forgotten here that the mainstream also claims the most distant galaxies are the oldest galaxies.

GN-108036 The redshift was $\mathrm{z}=7.2$, meaning the light of the galaxy took 12.9 billion years to reach Earth and therefore its formation dates back to 750 million years after the Big Bang Redshift $\mathrm{z}=7.213$.

GN-z11 $\approx 32$ billion ly (9.8 billion pc) (present proper distance); $\approx 13.4$ billion ly (4.1 billion pc) (light-travel distance); Helio radial velocity $295.050 \pm 119.917 \mathrm{~km} / \mathrm{s}$. 


\begin{tabular}{llll}
\hline M33 & $-0,000607(\mathrm{z})$ & $2,38-3,07$ Mly distance & $-179 \pm 3 \mathrm{~km} / \mathrm{s}$ \\
$\underline{\text { M64 }}$ & 0,001361 (z) & $24 \pm 7$ Mly & $408 \pm 4 \mathrm{~km} / \mathrm{s}$ \\
$\underline{\text { CID-42 }}$ & $0,359 \quad(\mathrm{z})$ & 3,9 Gly & $89.302 \mathrm{~km} / \mathrm{s}$ \\
$\underline{\text { MS 1054-03 }}$ & 0,8321 (z) & 6,757 Gly & $246.759 \mathrm{~km} / \mathrm{s}$
\end{tabular}

So, what is correct here: that the most distant galaxies withdraw at the fastest speed, or that the oldest galaxies had been withdrawing at the fastest speed?

If the most distant galaxies are at the same time the oldest, then the fastest were the galaxies in the far past, so-called protogalaxies - and that is opposite to the claim that Universe spreads ever faster.

The next table shows that radiation incoming from the distances of more than 12 Gly from all directions of the volume of Universe are measured.

Table 4. The Direction of the Farthest Galaxies within the Universe

\begin{tabular}{|c|c|c|c|c|c|}
\hline & Galaxy & Right ascension & Declination & Red shift & Distance G ly \\
\hline 1 & HCM-6A & $02^{\mathrm{h}} 39^{\mathrm{m}} 54.7^{\mathrm{s}}$ & $-01^{\circ} 33^{\prime} 32^{\prime \prime}$ & 6,56 & 12,8 \\
\hline 2 & $\overline{\text { SXDF-NB } 1006-2}$ & $02^{\mathrm{h}} 18^{\mathrm{m}} 56.5^{\mathrm{s}}$ & $-05^{\circ} 19^{\prime} 58.9^{\prime \prime}$ & 7,215 & 13,07 \\
\hline 3 & TN J0924-2201 & $09^{\mathrm{h}} 24^{\mathrm{m}} \quad 19,92^{\mathrm{s}}$ & $-22^{\circ} 01 ' 41,5 "$ & 5,19 & 12,523 \\
\hline 4 & $\overline{\text { UDFy-38135539 }}$ & $03^{\mathrm{h}} 32^{\mathrm{m}} 38.13^{\mathrm{s}}$ & $-27^{\circ} 45^{\prime} 53.9^{\prime \prime}$ & 8,6 & 13,1 \\
\hline 5 & A2744 YD4 & $00^{\mathrm{h}} 14^{\mathrm{m}} 24.927^{\mathrm{s}}$ & $-30^{\circ} 22^{\prime} 56.15^{\prime \prime}$ & 8,38 & 13,2 \\
\hline 6 & $\overline{\mathrm{BDF}-3299}$ & $22^{\mathrm{h}} 28^{\mathrm{m}} 12.26^{\mathrm{s}}$ & $-35^{\circ} 09^{\prime} 59.4^{\prime \prime}$ & 7,109 & 13,05 \\
\hline 7 & SSA22-HCM1 & $22^{\mathrm{h}} 17^{\mathrm{m}} 39.69^{\mathrm{s}}$ & $+00^{\circ} 13^{\prime} 48.6^{\prime \prime}$ & 5,47 & 12,7 \\
\hline 8 & EQ J100054+023435 & $10^{\mathrm{h}} 00^{\mathrm{m}} 54.52^{\mathrm{s}}$ & $+2^{\circ} 34^{\prime} 35.17^{\prime \prime}$ & 4,547 & 12,2 \\
\hline 9 & ULAS J1120+0641 & $11^{\mathrm{h}} 20^{\mathrm{m}} 01.48^{\mathrm{s}}$ & $+06^{\circ} 41^{\prime} 24.3^{\prime \prime}$ & 7,085 & 13,05 \\
\hline 10 & ULAS J1342+0928 & $13^{\mathrm{h}} 42^{\mathrm{m}} 08.10^{\mathrm{s}}$ & $+13^{\mathrm{h}} 42^{\mathrm{m}} 08.10^{\mathrm{s}}$ & 7,54 & 13,1 \\
\hline 11 & GRB 090423 & $09^{\mathrm{h}} 55^{\mathrm{m}} 33.08^{\mathrm{s}}$ & $+18^{\circ} 08^{\prime} 58.9^{\prime \prime}$ & 8,2 & 13 \\
\hline 12 & $\overline{\mathrm{IOK}-1}$ & $13^{\mathrm{h}} 23^{\mathrm{m}} 59.8^{\mathrm{s}}$ & $+27^{\circ} 24^{\prime} 56^{\prime \prime}$ & 6,96 & 12,88 \\
\hline 13 & A1703 zD6 & $13^{\mathrm{h}} 15^{\mathrm{m}} 01.0^{\mathrm{s}}$ & $+51^{\circ} 50^{\prime} 04^{\prime}$ & 7,054 & 13,04 \\
\hline 14 & $\mathrm{Q} 0906+6930$ & $09^{\mathrm{h}} 06^{\mathrm{m}} 30.75^{\mathrm{s}}$ & $+69^{\circ} 30^{\prime} 30.8^{\prime \prime}$ & 5,47 & 12,3 \\
\hline 15 & MACS0647-JD & $06^{\mathrm{h}} 47^{\mathrm{m}} 55.73^{\mathrm{s}}$ & $+70^{\circ} 14^{\prime} 35.8^{\prime \prime}$ & 10,7 & 13,3 \\
\hline
\end{tabular}

Table 4. The direction of the farthest galaxies within the Universe distance 12,2 -13,3 G ly

(Duckss, 2019)

The table shows galaxies from $00 \mathrm{~h} 14 \mathrm{~m} 24.927 \mathrm{~s}$ to $22 \mathrm{~h} 28 \mathrm{~m} 12.26 \mathrm{~s}$ equatorial and $-35^{\circ}$ $09^{\prime} 59.4^{\prime \prime}$ to $+70^{\circ} 14^{\prime} 35.8^{\prime \prime}$ to the north/south from the celestial equator. Namely the measurements of galactic distances, advocated by the mainstream, indicate that similar distances are measured in all directions. These measurements represent the volume of Universe as being opposite to their claims of total maximum age of Universe of 13,8 Gly. The forms of radiation (measured recently) above 12 Gly approach from all parts of the volume.

Now, from the table that recalculates real distances above $5(\mathrm{z})$ and less into Big Bang constructs, it is again obvious that the diameter of Universe is twice as big as 13,8 Gly. When "real" values of correct interpretation of redshift are included

(With a redshift of 5.47,(Harvey, 2015)(NASA/IPAC , 2010) (Q0906 + 6930) light from this active galaxy is estimated to have taken around 12.3 billion light-years to reach us.. distance to this galaxy is estimated to be around 26 billion light-years (7961 Mpc). (Wikipedia) there is another problem. 12,3 billion light-years multiplied with 2 makes 24,6 billion light-years, which is by 1,4 Gly less, if a limiting condition that radiation and expansion have been moving at the same speed is taken into consideration. The same difference continues to grow when (z) grows:

ULAS J1120+0641 Redshift 7.085 \pm 0.003(Harvey, 2015); distance 28.85 Gly (8.85 Gpc) 
(co-moving) (NASA/IPAC, 2010); 12.9 Gly (4.0 Gpc) (light travel distance), the difference is 3,05 ,

UDFy-38135539 (z) 8,6; The light travel distance of the light that we observe from UDFy-38135539 (HUF.YD3) is more than 4 billion parsecs(Duckss, 2013) (13.1 billion light years), and it has a luminosity distance of 86.9 billion parsecs (about 283 billion light years), the difference is 270 Gly.

Here, the data should also be included, that for dist. 2.4 Gly we measure red shift (z) 0,211 (Abell_222(3); za dist. 12,0 Gly we measure (z) 1,26, 1,27 (Lynx Supercluster) and other data from Table 3.

The galaxy GN-z11 dist. 13,39 Gly has (z) 11,09 and it has a more significant redshift by 10,63 (4) than Lynx Supercluster $(1,26(7) 12.9$ billion light years) but the distance is larger only by 0,49 Gly.

For the distance of 0,7 Gly Musket Ball Cluster there is a value of 0,53(z), while the difference here is 10,63 Gly. The difference of (z) 10,63 matches Abell 1835 IR1916 which has (z) 10 and recommended age (distance) of 13,2 Gly.

Let us repeat that:

„The proper distance for a redshift of 8.2 would be about $9.2 \mathrm{Gpc}$, or about 30 billion light years.“ „With a redshift of 5.47, (Q0906 + 6930) light from this active galaxy is estimated to have taken around 12.3 billion light-years to reach us.. distance to this galaxy is estimated to be around 26 billion light-years (7961 Mpc). (Attila, 2009)

The rotation of Universe (instead of expansion) that is based on the similar principles as the rotation of clusters of galaxies or stars, is also unable to accept such confused data, because there are no very significant deviations by the volume of cluster. The internal galaxies move slower than the external ones, but make a single orbital cycle approximately at the same time. Under these conditions the measured value of redshift $(\mathrm{z})$ and current distance between the measured objects are approximately the same. In the case Universe would be rotating, its diameter is presented in the table 4 and if the definition of redshift value, according to the mainstream, is used, the diameter goes above 25 Gly.

When including the decrease of wave intensity (with the increase of speed, currently used by the mainstream) as a dominant value in determining distances of objects in Universe, it would completely remove the existence of two values of interpreting distance or Comoving distance- light travel distance. Also, the obstacles to calculate real values of redshift would be gone. Very large quantity of objects (measured recently) will be the part of the volume of our Universe, a part of them will be waves incoming from the neighboring universes (our local group of universes).

„By applying the analogy of the ascending sequence of events, the more we are distanced from the source of radiation, the lower are the temperatures. Between the multi-universes, they are a bit closer to the absolute zero. The temperatures decrease as the wholes grow. An endlessly large volumetric belt of energy is expanding after the last ascending whole and the temperature there is absolute zero.

By the analogy, inside this belt there is an endless quantity of the wholes, similar to that one, but it is very likely that the whole with the absolute zero temperature in it could be the outer and the last whole in the hierarchy that goes further into the 3-D infinity (at least the infinity as humankind understands it).,, (Duckss, 2013) 


\section{Conclusion}

With the transited distance, waves lose their intensity that is registered by the increasing redshift (Mean Solar Irradiance (W/m2) on Mercury is 9.116,4, Earth 1.366,1, Jupiter 50,5, na Pluto 0,878 (Duckss, 2018)).

Confronting the evidence, that in recent time there are 200000 of merging or colliding galaxies and have blueshift among themselves, some people are persistent in continuing that the increase of the galactic speed exclusively affects the value of redshift. A significant sum of evidence states there is also a redshift in those galaxies that are approaching to an observer (but only those that are further than 70 Mly away, while those that are closer than that register a blueshift).

By continuing to use such a platform, unbelievable fabrications occur, which have no place in physics. They distort real measurements and instead of science they try to incorporate into physics some "values" that do not belong to it.

Measurements should be presented exclusively within realistic values and there is no need for subsequent embellishments to preserve such structures that exceed the limits of physics.

It is necessary to determine real values of the influence of the radiation intensity weakening (with a constant and slow increase of speed of the distant galaxies in their orbits inside Universe) within the redshift value. The clusters of galaxies have rotations that are different from zero and due to their constant orbital rotation it seems to an observer that galaxies have very different directions of movement. Generally, they travel in the orbit of their cluster, as a dot on a planet, a star that rotates together with its planet inside their galaxy, which rotates further in its cluster and that cluster within its supercluster and finally all together rotate in Universe.

\section{References}

Attila, M. (2009). "Impact on cosmology of the celestial anisotropy of the short gamma-ray bursts". Baltic Astronomy.

https://en.wikipedia.org/wiki/Observable_universe\#Most_distant_objects.

Duckss, W. (2018). Demolition Hubbles law big bang the basis of modern and ecclesiastical cosmology. http://www.globalscientificjournal.com/researchpaper/Demolition-hubbleslaw-big-bang-the-basis-of-modern-and-ecclesiastical-cosmology.pdf Volume 6, Issue 3, March 2018, GSJ@ 2018 www.globalscientificjournal.com

Duckss, W. (2018). How are the spiral and other types of galaxies formed? American Journal of Astronomy and Astrophysics.

http://www.sciencepublishinggroup.com/journal/paperinfo?journalid=301\&doi=10.116 48/j.ajaa.20180603.13

Duckss, W. (2019). The Processes of Violent Disintegration and Natural Creation of Matter in the Universe. Budapest International Research in Exact Sciences (BirEx) Journal. https://bircu-journal.com/index.php/birex/article/view/474

Duckss, W. (2019). Effects of Rotation Arund the Axis on the Stars, Galaxy and Rotation of Universe. https://www.ijsciences.com/pub/pdf/V82019021908.pdf, Weitter Duckss.

Duckss, W. (2019). Collaboration Robots with Artificial Intelligence (AI) as Digital Doubles of Person for Communication in Public Life and Space, Evgeniy Bryndin (Russia), Budapest International Research in Exact Sciences (BirEx) Journal

Duckss, W. (2016). The observation process in the universe through the database. Slavko Sedic. International Journal of Scientific \& Engineering Research, Volume 7, Issue 10, 
October-2016 408. http://www.ijser.org/onlineResearchPaperViewer.aspx?Theobservation-process-in-the-universe-through-the-database.pdf , (W.Duckss)

Duckss, W. (2013). Why is the universe cold? https://www.svemir-ipaksevrti.com/theUniverse-rotating.html\#15b 2013/14.

Harvey, D. et al (2015). The non-gravitational interactions of dark matter in colliding galaxy clusters.https://www.spacetelescope.org/static/archives/releases/science_papers/heic150 6a.pdf 2015.

NASA/IPAC. (2010). Extragalactic Database" Results for 0901+6942. Retrieved 2010-0420.https://en.wikipedia.org/wiki/Q0906\%2B6930\#Distance_measurements.

Samuel. (2019). On the Performance of Transmuted Logistic Distribution: Statistical Properties and Application, Adeyinka Femi Samuel, Budapest International Research in Exact Sciences (BirEx) Journal. https://bircujournal.com/index.php/birex/article/view/341 Vol 1, No 3 (2019) > 